Mon. Not. R. Astron. Soc. 000, 1-12 (2009) Printed 5 September $2021 \quad$ (MN LATEX style file v2.2)

\title{
Multi-epoch intra-night optical monitoring of 8 radio-quiet BL Lac candidates
}

\author{
P. Kumar ${ }^{1 \star}$, Gopal-Krishna $^{2}$, C. S. Stalin ${ }^{3}$, H. Chand ${ }^{1}$, R. Srianand ${ }^{4}$, P. Petitjean ${ }^{5}$ \\ ${ }^{1}$ Aryabhatta Research Institute of Observational Sciences (ARIES), Manora Peak, Nainital, 263002 India \\ ${ }^{2}$ Centre for Excellence in Basic Sciences (CEBS), University of Mumbai campus (Kalina), Mumbai 400098, India \\ ${ }^{3}$ Indian Institute of Astrophysics, Block II, Koramangala, Bangalore-560034, India \\ ${ }^{4}$ Inter-University Centre for Astronomy and Astrophysics (IUCAA), Postbag 4, Ganeshkhind, Pune 411 007, India \\ ${ }^{5}$ UPMC-CNRS, UMR7095, Institut d'Astrophysique de Paris, F-75014 Paris, France
}

Accepted - . Received —; in original form -

\begin{abstract}
For a new sample of 8 weak-line-quasars (WLQs) we report a sensitive search in 20 intranight monitoring sessions, for blazar-like optical flux variations on hour-like and longer time scale (day/month/year-like). The sample consists exclusively of the WLQs that are not radio-loud and have either been classified as 'radio-weak probable BL Lac candidates' and/or are known to have exhibited at least one episode of large, blazarlike optical variability. Whereas only a hint of intra-night variability is seen for two of these WLQs, J104833.5+620305.0( $z=0.219)$ and J133219.6+622715.9 ( $z=3.15)$, statistically significant inter-night variability at a few per cent level is detected for three of the sources, including the radio-intermediate WLQ J133219.6+622715.9 $(z=3.15)$ and the well known bona-fide radio-quiet WLQs J121221.5+534128.0 $(z=3.10)$ and WLQ J153259.9-003944.1 $(\mathrm{z}=4.62)$. In the rest-frame, this variability is intra-day and in the far-UV band. On the time scale of a decade, we find for three of the WLQs large brightness changes, amounting to $1.655 \pm 0.009,0.163 \pm 0.010$ and $0.144 \pm 0.018$ mag, for J104833.5+620305.0, J123743.1+630144.9 and J232428.4+144324.4, respectively. Whereas the latter two are confirmed radio-quiet WLQs, the extragalactic nature of J104833.5+620305.0 remains to be well established, thanks to the absence of any feature(s) in its available optical spectra. The present study forms a part of our ongoing campaign of intranight optical monitoring of radio quiet weak-line quasars, in order to improve the understanding of this enigmatic class of Active Galactic Nuclei and to look among them for a possible tiny, elusive population of radio-quiet BL Lacs.
\end{abstract}

Key words: galaxies: active — quasars: emission lines - quasars: general Bl-Lacertae objects: general — galaxies: photometry — galaxies: jets.

\section{INTRODUCTION}

Being among the most active extragalactic sources, the radio-to-optical/UV observations of BL Lacs are marked by strong and rapid variability of both continuum and polarized emission, commonly attributed to the dominance of a relativistically boosted jet of synchrotron radiation, emanating from the core of the host galaxy (e.g., Blandford \& Rees 1978). Possibly, the same jet dominance effectively contributes to the weak appearance (or, non-detection) of the emission lines in the optical/UV spectrum, which is a defining characteristic of the BL

\footnotetext{
* E-mail: parveen@aries.res.in (PK)
}

Lac objects (e.g., Angel \& Stockman 1980; Begelman et al. 1984; Urry \& Padovani 1995; Antonucci 2012, and references therein). The availability of spectra of thousands of faint, star-like objects covered in the large imaging/spectroscopic surveys, such as the Sloan Digital Sky Survey (SDSS; e.g., York et al. 2000; Schneider et al. 2005) and the Two-Degree Field QSO Redshift Survey (2QZ; Boyle et al. 2000; Croom et al. 2001) paved the way for making systematic optical searches for BL Lac objects, which had been previously found mainly by an optical follow-up of the candidates first detected in the radio or X-ray bands (e.g., Stickel et al. 1991; Stocke et al. 1991; Perlman et al. 1996; Padovani et al. 2007; Anderson et al. 2007, and references therein). Although, on a limited scale, 
optical variability on month/year-like time scales has also been employed as a technique for finding AGN candidates (e.g., Villforth et al. 2010; Bauer et al. 2009, and references therein), the big boost in this direction came with the advent of massive optical surveys. Indeed, these optical surveys began to reveal in large numbers distant, high-luminosity objects which qualified as potential BL Lacs by virtue of their stellar appearance and an essentially featureless optical spectrum. These objects were termed 'weak-line-quasars' (WLQs) (e.g., Fan et al. 1999; Anderson et al. 2001; Londish et al. 2002; Collinge et al. 2005; Schneider et al. 2005; Fan et al. 2006; Londish et al. 2007; Plotkin et al. 2008; Diamond-Stanic et al. 2009). It was even suggested that the WLQs could be the long sought high-z counterparts of BL Lacs (e.g., Shemmer et al. 2006; Stocke 2001). However, the radio and X-ray outputs as well as the strength and variability of both continuum and polarized emission of most WLQs are generally modest, in a striking contrast to BL Lac objects, putting a question mark on their BL Lac classification (e.g., Meusinger \& Balafkan (2014) and references therein; Plotkin et al. (2015); Luo et al. (2015); see below).

The combination of huge size and depth of the optical surveys has also revived interest in the old question whether a rare population of BL Lacs exists which is radio-quiet, in analogy to the radio-quiet quasars (radioquietness is usually parameterised by the ratio of radio and optical flux densities, $\mathrm{R}^{1}$. Attempts to discover radioquiet BL Lacs, previously triggered by the large X-ray surveys (e.g., Chanan et al. 1982; Stocke et al. 1991) found little substantiation from the follow-up optical polarimetry of the BL Lac candidates gleaned from the X-ray surveys (e.g., Borra \& Corriveau 1984; Jannuzi et al. 1993). A similar lack of confirmation emerged from the polarimetric follow-up of the radio-quiet BL Lac candidates extracted from the various optical surveys including the massive surveys mentioned above (see, e.g., Impey \& Brand 1982; Stocke et al. 1990; Smith et al. 2007; Heidt \& Nilsson 2011, and references therein). Likewise, the broad-band spectral measurements and optical monitoring to detect continuum variability on month/year-like time scales have shown that radio-quiet WLQs (RQWLQs) are mostly distinct from BL Lacs (e.g., Diamond-Stanic et al. 2009; Lane et al. 2011; Wu et al. 2012; Meusinger \& Balafkan 2014; Plotkin et al. 2015; Kügler et al. 2015). A similar inference is reached when X-ray properties of high-z WLQs are examined (Shemmer et al. 2009).

A number of proposals have been put forward to explain the uncharacteristically weak line emission from WLQs. In one scheme, the weakness has been attributed to a deficit of gas in the broad emission-line region (BELR) of WLQs, either because the quasar activity has begun only recently (Hryniewicz et al. 2010; Murray \& Chiang 1997), or due to some other possible cause (e.g., Shemmer et al. 2010). A competing explanation (e.g., Nikołajuk \& Walter 2012) argues that the BELR in WLQs has a very low covering factor against the flux of ionizing photons in-

1 Radio-loudness is usually parametrized by the ratio ( $R$ ) of flux densities at $5 \mathrm{GHz}$ and at $4400 \AA$ in the rest-frame, and $\mathrm{R}<10$ for radio-quiet quasars (e.g. see, Kellermann et al. 1989). cident from the accretion disk. Possibly, this situation could arise, for instance, due to a shielding gas layer (or, patchy shielding gas) between the hot accretion disk and the BELR, which attenuates the flux of ionizing photons emerging from the accretion disk (Lane et al. 2011; Wu et al. 2011, 2012); also, (Luo et al. 2015). Alternative explanations implicate the physics of disk accretion onto the central super-massive black-hole (e.g., Laor \& Davis 2011; Leighly et al. 2007; Nicastro et al. 2003). While all these theoretical scenarios are debated, substantial evidence has accumulated showing that the central engines of at least some radio-quiet quasars 'RQQs' (including their weak line counterparts, the RQWLQs) are in fact capable of ejecting relativistic jets which are mostly feeble and extended on just the parsec scale. This inference is rooted in the radio continuum imaging including VLBI and flux monitoring campaigns targeted at RQQs (e.g., Blundell \& Beasley 1998; Barvainis et al. 2005; Caccianiga et al. 2001; Ulvestad et al. 2005; Falcke et al. 1996; Leipski et al. 2006; Herrera Ruiz et al. 2016). It is also interesting to recall the striking discovery of an intermittently present, relativistically beamed nuclear jet of radio and X-ray emission (Blundell et al. 2003; Gallo 2006) in the $z=0.94$ WLQ PG 1407+265 (J140923.9+261821.1; (McDowell et al. 1995), which has been classified as 'radioquiet'. Likewise, the possibility of a relativistic jet in the $z$ $=4.62$ RQWLQ J153259.96-003944.1 has been inferred on the basis of its strong optical variability (Stalin \& Srianand 2005). However, the significance and importance of these findings remains to be consolidated via polarimetric monitoring of the RQWLQs short-listed as radio-quiet BL Lac candidates, since a high and variable polarization is the hallmark of bona-fide BL Lacs. A robust finding of even a minuscule population of radio-quiet BL Lacs, which could well be lurking in samples of RQWLQs (e.g., see, Collinge et al. 2005; Shemmer et al. 2006; Lane et al. 2011; $\mathrm{Wu}$ et al. 2012), would provide an important new insight into the nature of AGN jets. For instance, a remarkable ramification would be that in some rare relativistic jets, either the synchrotron radio emission is abnormally weak intrinsically, or the relativistic Doppler boost somehow is uncharacteristically ineffective in the radio domain than at optical wavelengths. It is, however, clear that if at all such exotic objects exist, they must be extremely rare, as suggested by the afore-mentioned studies of WLQs employing a number of basic diagnostics, such as the multi-wavelength spectral/polarimetric measurements, and optical flux variability, all of which seem to favor the notion that at least an overwhelming majority of RQWLQs is different from BL Lacs, whether radio or X-ray selected (e.g., Diamond-Stanic et al. 2009; Shemmer et al. 2009; Lane et al. 2011; Kügler et al. 2014a; Meusinger \& Balafkan 2014; Plotkin et al. 2015). This inference about rarity of jet emission in RQWLQs is further supported by the frequently observed deficit of $\mathrm{X}$ ray emission, though it is somewhat unclear how much of it is caused due to absorption (see, e.g., Luo et al. 2015).

In a parallel attempt aimed at finding radio-quiet BL Lac candidates, we initiated a program to search for rapid optical variability (on hour-like time scale) in a few dozen RQWLQs which had shown some signatures of potential radio-quiet BL Lacs. The candidates were selected from the catalogs of WLQs published by Plotkin et al. (2010b) and 
Meusinger \& Balafkan (2014). The results obtained so far under this program to search for intra-night optical variability (INOV) in 25 radio-quiet BL Lac candidates, monitored by us in 48 sessions of minimum 3 hour duration, have been reported in 4 papers ( Gopal-Krishna et al. (2013): Paper I, Chand et al. (2014): Paper II, Kumar et al. (2015): Paper III, Kumar et al. (2016): Paper IV). Only two events of INOV were detected, out of the 48 monitoring sessions, corresponding to an INOV duty cycle of $\sim 3 \%$, which is much lower than that known for BL Lacs (e.g., Goyal et al. 2013). However, on both occasions the observed INOV had a large amplitude ( $>10 \%)$, hinting at a blazar-like behavior for the two RQWLQs, namely, J090843.25+285229.8 (Paper II) and J140710.26+241853.6 (Paper III). Conceivably, the inferred smaller duty cycle could be due to the lack of a matching sensitivity for monitoring WLQs which are mostly fainter in comparison to the classical BL Lacs that have typically been covered in intranight monitoring programs (e.g., Papers III and IV). In this paper, we present results from 20 sessions of intranight optical monitoring (using 1 to 2 -meter class telescopes), covering a new set of 8 WLQs which are not radio-loud (Table 1 ). Note that all these objects, excepting J140923.9+261821.1, had also been monitored under a separate program nearly a decade ago by some of the present authors and those independently taken unpublished observations have been included in the present work.

\section{THE SAMPLE SELECTION}

Six out of the 8 WLQs in the present sample are drawn from the list of 27 "Potential radio-weak BL Lac candidates", derived by Collinge et al. (2005)) from the SDSS DR2 (Abazajian et al. 2004) and the 2DF survey (Boyle et al. 2000), by imposing the criteria of radioquietness (Table 1; see also Kellermann et al. 1989), a nearly featureless optical spectrum (i.e., no line with an EW $>5 \AA$ in the rest-frame), lack of proper motion above $2.5 \sigma$ threshold and a broad-band color criterion $(g-r \geqslant 0.35$ or $r-i \geqslant 0.13$ ) (Collinge et al. 2005) which is expected to minimize contamination by Galactic stars, such as DC white dwarfs. Application of some additional selection criteria consistent with BL Lacs led them to the list of 27 sources. These additional criteria are: (i) absence of stellar absorption features at zero redshift, (ii) a spectrum inconsistent with the stellar template, or combination of galaxy Eigen spectra, (iii) the strength of $\mathrm{Ca}$ II H \& $\mathrm{K}$ break being $<0.4$. Out of the 27 candidate radio-quiet BL Lacs, we extracted a representative set of six without any bias towards optical variability, or redshift, such that our target selection was primarily dictated by the availability of the telescope time, scheduling considerations and the availability of at least two, but usually more, comparison stars on each CCD frame, within about 1 mag of the target.

An independent check was made with the catalog of Monet et al. (2003), to ensure that all our sources are consistent with having zero proper motion(Table 1). The remaining two WLQs in our sample are PG $1407+265(z=$ 0.94 ) and J153259.96-003944.1 ( $z=4.62)$. Both these well known radio-quiet WLQs are reported to have shown an episode of blazar-like strong optical flaring (Section 1). The basic properties of our set of 8 WLQs are summarized in Table 1.

\section{OBSERVATION AND REDUCTION}

The photometric observations were carried out in the R-band using the 2.0-m Himalayan Chandra Telescope (HCT) of the Indian Astronomical Observatory (IAO) at Hanle (Prabhu \& Anupama 2010), the 1.3-m Devasthal Fast Optical Telescope (DFOT) located near Nainital (India) (Sagar et al. 2011) and the 1.2-m telescope of the Observatoire de Haute-Provence (OHP), France ${ }^{2}$.

The 2.0-m HCT has a Ritchey-Chretien (RC) design with a f/9 beam at the Cassegrain focus. The detector used is a cryogenically cooled $2048 \times 4096$ chip, having a pixel size of 15 micron and a plate scale of 0.29 arcsec per pixel which covers an area of $\sim 10 \mathrm{arcmin}$ on the sky. The readout noise of the CCD is $4.8 \mathrm{e}-/$ pixel the gain is $1.22 \mathrm{e}-/$ Analog to Digital Unit (ADU).

The 1.3-m DFOT is a fast beam (f/4) optical telescope with a pointing accuracy better than $10 \operatorname{arcsec}(\mathrm{rms})$. It is equipped with a $512 \mathrm{k} \times 512 \mathrm{k}$ Andor CCD camera having a pixel size of 16 micron and a plate scale of 0.63 arcsec per pixel. The CCD covers a field of view of $\sim 5$ arcmin on the sky. It is cooled thermo-electrically to $-90 \operatorname{degC}$ and is read out at $1 \mathrm{MHz}$ speed. The corresponding system noise is 6.1 e- (rms) and the gain is $1.4 \mathrm{e}-/ \mathrm{ADU}$.

The 1.2-m OHP telescope is a f/6 optical telescope with Newtonian focus. The detector is a thin backilluminated $1024 \times 1024$ Tektronix chip, with a read-out noise of 8.5 e- and a gain of $3.5 \mathrm{e}-/ \mathrm{ADU}$. This instrument provides a field of view of $\sim 11.7$ arcmin on the sky with a plate scale of 0.68 arcsec per pixel.

The exposure time for each science frame was set between 5 and 30 minutes, typically yielding a signal-to-noise ratio above $25-30$. The typical seeing (FWHM) during our observations was around 2 arcsec. Since in the sample selection, care was taken to ensure the availability of at least two, but usually more, comparison stars on each CCD frame, within about $1 \mathrm{mag}$ of the target WLQ, it became possible to identify and discount any comparison star(s) which showed a hint of variability during a given monitoring session. Although we have endeavored to take comparison stars that are close to the monitored AGN, both in brightness and color, a deviation from this became necessary sometimes when trying to compare Differential Light Curves (DLCs) of the same AGN taken on different nights. This happened because of our insistence on using for a given AGN the same set of comparison stars on all the nights, even if different telescopes (having unequal fields-of-view) had been used on those nights. This has occasionally led to a situation when the DLCs used for the purpose of INOV and for 'short-term optical variability (STOV) or 'long-term optical variability (LTOV) employ different comparison stars for the same AGN (see, Table 2).

The pre-processing of the raw CCD images (bias subtraction, flat-fielding, cosmic-ray removal and trimming) was 
Table 1. Basic parameters of the present sample of 8 WLQs

\begin{tabular}{|c|c|c|c|c|c|c|c|}
\hline $\begin{array}{l}\text { Name } \\
\text { (1) }\end{array}$ & $\begin{array}{l}\text { R.A.(J2000) } \\
\qquad \begin{array}{c}(\mathrm{h} \mathrm{m} \mathrm{s}) \\
(2)\end{array}\end{array}$ & $\begin{array}{c}\text { DEC. }(\mathrm{J} 2000) \\
\left(\begin{array}{l}0 \prime \prime \prime \\
\prime \prime\end{array}\right) \\
(3)\end{array}$ & $\begin{array}{c}\mathrm{r}(\mathrm{mag}) \\
(4)\end{array}$ & $\begin{array}{l}\mathrm{z}^{a} \\
(5)\end{array}$ & $\begin{array}{c}\text { Proper Motion } \\
\text { (mas/yr) } \\
(6)\end{array}$ & $\begin{array}{c}\alpha_{r-o}{ }^{c} \\
(7)\end{array}$ & $\begin{array}{c}\text { Telescope } \\
\text { (8) }\end{array}$ \\
\hline $\mathrm{J} 104833.5+620305.0^{d}$ & $10: 48: 33.56$ & $+62: 03: 05.0$ & 19.86 & $0.219 \pm 0.178^{\dagger}$ & $14.14 \pm 7.07$ & $<0.27^{\star}$ & $\mathrm{HCT}$ \\
\hline $\mathrm{J} 121221.5+534128.0$ & $12: 12: 21.56$ & $+53: 41: 28.0$ & 18.63 & $3.0976 \pm 0.0003^{*}$ & 0 & $<0.03^{\star}$ & $\mathrm{OHP}$ \\
\hline $\mathrm{J} 123743.1+630144.9$ & $12: 37: 43.14$ & $+63: 01: 44.9$ & 19.00 & $3.4278 \pm 0.0023^{*}$ & 0 & $<0.04^{\star}$ & OHP \\
\hline $\mathrm{J} 124225.3+642919.0$ & $12: 42: 25.39$ & $+64: 29: 19.0$ & 15.69 & $0.04247 \pm 0.00002^{\dagger}$ & 0 & $<-0.06^{\ddagger}$ & $\mathrm{OHP}$ \\
\hline $\mathrm{J} 133219.6+622715.9$ & $13: 32: 19.65$ & $+62: 27: 15.9$ & 19.19 & $3.1514 \pm 0.0023^{*}$ & $8.94 \pm 3.61$ & $0.19^{\star}$ & $\mathrm{HCT}$ \\
\hline $\mathrm{J} 140923.9+261821.1$ & $14: 09: 23.91$ & $+26: 18: 21.1$ & 15.74 & $0.94 \pm 0.02$ & $6.32 \pm 4.472$ & $0.10^{*}$ & DFOT \\
\hline J153259.9-003944.1 & $15: 32: 59.96$ & $-00: 39: 44.1$ & 21.16 & $4.62 \pm 0.04$ & 0 & $<0.02^{\$}$ & $\mathrm{HCT}$ \\
\hline $\mathrm{J} 232428.4+144324.4$ & $23: 24: 28.43$ & $+14: 43: 24.4$ & 18.79 & $1.4172 \pm 0.0006^{*}$ & 0 & $<0.01^{\ddagger}$ & $\mathrm{HCT}$ \\
\hline
\end{tabular}

${ }^{a}$ Reference is Hewett \& Wild (2010) for *; SDSS catalog for ${ }^{\dagger}$; McDowell et al. (1995) for J140923.9+261821.1. and Fan et al. (1999) for J153259.9-003944.1

$b$ Monet et al. (2003).

$c$ The radio-optical spectral index $\alpha_{r-o}$ is usually taken to be less than 0.21 (e.g., Luo et al. 2015) for radio-quiet quasars having radio-loudness parameter $R<10$, where $\mathrm{R}$ is the ratio of flux densities at $5 \mathrm{GHz}$ and at $4400 \AA$ in rest frame (e.g., Kellermann et al. 1989). Reference for $\alpha_{r-o}$ is Collinge et al. (2005) for *; Plotkin et al. (2010a) for $\ddagger$; Fan et al. (1999) for $\$$ and Kellermann et al. (1989) for $*$.

$d$ The extragalctic nature of this source is uncertain (e.g., see, Section 5.1) as it has been classified in the SDSS as a star. Richards et al. (2009) give its photometric redshift as 1.54.

done using the standard tasks available in the Image Reduction and Analysis Facility (IRAF) ${ }^{3}$. The instrumental magnitudes of the observed target AGN and the chosen comparison stars in each CCD frame were determined by aperture photometry (Stetson 1992, 1987), using the Dominion Astronomical Observatory Photometry II (DAOPHOT II algorithm $)^{4}$. To select the aperture size (FWHM) for photometry, we first determined the "seeing" for each frame by averaging the observed FWHMs of 5 moderately bright stars in the CCD frame. We then took the median of these averaged values over all the frames recorded in the session. The aperture diameter was set equal to 2 times the median FWHM. The only exception to this procedure is the nearby WLQ J124225.3+642919.0 $(z=0.04247)$ for which we chose a fixed aperture of 4 arcsec diameter in view of the fact that the underlying fuzz due to the host galaxy is quite prominent (see, Carini et al. 1991; Cellone et al. 2000). As shown below, this object did not exhibit significant variability, either on hour-like or longer time scale and this conclusion is not sensitive to the size of the aperture chosen.

To derive the DLCs of the target WLQ monitored in a given session, we selected two steady comparison stars which are present in all the CCD frames and are also close to the WLQ monitored, both in location and brightness. Particulars of the comparison stars used for the various sessions are given in Table 2. Note that with the solitary exception of the WLQ J1532-0039, the $g-r$ color difference between the target WLQ and its comparison stars is always within $\sim 1$-mag, with a median value of $0.6-\operatorname{mag}$ (Table 2). Analysis by Stalin et al. (2004a,b) has shown that for color difference of this order, the changing atmospheric attenuation

\footnotetext{
3 Image Reduction And Analysis Facility (HTTP://IRAF.NOAO.EDU/).

4 Dominion Astrophysical ObServatory Photometry.
}

during a session produces a negligible effect on the DLCs (see, also, Carini et al. (1992)) .

\section{STATISTICAL ANALYSIS}

C-statistic (e.g., Jang \& Miller 1997) has been the most commonly used and the one-way analysis of variance (ANOVA) (de Diego 2010) the most powerful test for verifying the presence of variability in a DLC. However, we did not employ either of these tests here because, de Diego (2010) has questioned the validity of the C-test, arguing that the C-statistics does not have a Gaussian distribution and the nominal critical value of 2.576 used for confirming the presence of variability at $3 \sigma$ level is usually too conservative. On the other hand, the ANOVA test requires a rather large number of data points in the DLC, so as to have several points within each sub-group used for the analysis. This is not feasible for our DLCs which typically have only about a dozen data points. So, we have instead used the $F$-test which is based on the ratio of variances, F = variance (observed)/variance(expected) (de Diego 2010; Villforth et al. 2010). Its two versions are: (i) the standard F-test (hereafter $F^{\eta}$-test, Goyal et al. (2012)) and (ii) scaled $F$-test (hereafter $F^{\kappa}$-test, Joshi et al. (2011)). The $F^{\kappa}$-test is preferred when the magnitude difference between the object and comparison stars is large (Joshi et al. 2011). Onward Paper II, we have only been using the $F^{\eta}$-test because our choosen comparison stars are usually quite comparable in brightness to the target AGN. An additional gain in using the $F^{\eta}$-test is that we can directly compare our INOV results with those now known for other major AGN classes (Goyal et al. 2013). An important point to keep in mind while applying the statistical tests is that the photometric errors on individual data points in a given DLC, as returned by the routine in the IRAF and DAOPHOT 
Weak emission line Quasars

Table 2. Basic parameters and observing dates for the 8 WLQs and their comparison stars monitored for INOV/STOV/LTOV (sect. 5).

\begin{tabular}{|c|c|c|c|c|c|c|c|c|c|c|c|c|}
\hline $\begin{array}{r}\text { IAU Name } \\
\text { (1) }\end{array}$ & $\begin{array}{c}\text { Date }^{a} \\
\text { dd.mm.yyyy } \\
(2)\end{array}$ & $\begin{array}{c}\text { R.A. (J2000) } \\
\left(\begin{array}{c}\text { h m s }) \\
(3)\end{array}\right.\end{array}$ & $\begin{array}{c}\text { Dec. }(\mathrm{J} 2000) \\
\left(\begin{array}{c}0, \prime \prime \\
(4)\end{array}\right.\end{array}$ & $\underset{(\mathrm{mag})}{\mathrm{g}}$ & $\begin{array}{c}\text { INOV } \\
\mathrm{r} \\
(\mathrm{mag}) \\
(6)\end{array}$ & $\begin{array}{c}\mathrm{g}-\mathrm{r} \\
(\mathrm{mag}) \\
(7)\end{array}$ & $\begin{array}{c}\mathrm{g} \\
(\mathrm{mag}) \\
(8)\end{array}$ & $\begin{array}{c}\text { STOV } \\
\text { r } \\
(\mathrm{mag}) \\
(9)\end{array}$ & $\begin{array}{c}\text { g-r } \\
(\mathrm{mag}) \\
(10)\end{array}$ & $\begin{array}{c}\mathrm{g} \\
(\mathrm{mag})\end{array}$ & $\begin{array}{c}\text { LTOV } \\
\text { r } \\
(\mathrm{mag}) \\
(12)\end{array}$ & $\begin{array}{c}\mathrm{g}-\mathrm{r} \\
(\mathrm{mag}) \\
(13)\end{array}$ \\
\hline $\begin{array}{r}\mathrm{J} 104833.5+620305.0 \\
\mathrm{~S} 1(\mathrm{INOV}) \\
\mathrm{S} 2(\mathrm{INOV})\end{array}$ & $\begin{array}{l}02.12 .2005 \\
02.12 .2005\end{array}$ & $\begin{array}{lll}10 & 48 & 33.56 \\
10 & 48 & 05.36 \\
10 & 48 & 35.90\end{array}$ & $\begin{array}{lll}+62 & 03 & 05.0 \\
+62 & 05 & 19.1 \\
+61 & 59 & 29.9\end{array}$ & $\begin{array}{l}20.22 \\
21.31 \\
20.63\end{array}$ & $\begin{array}{l}19.85 \\
19.57 \\
19.52\end{array}$ & $\begin{array}{l}0.37 \\
1.74 \\
1.11\end{array}$ & & & & & & \\
\hline S3(LTOV) & $02.12 .2005^{\dagger}, 29.04 .2016$ & $1048 \quad 52.36$ & $+6202 \quad 27.5$ & & & & & & & 18.52 & 17.84 & 0.68 \\
\hline S4(LTOV) & $02.12 .2005^{\dagger}, 29.04 .2016$ & 104833.90 & +620406.5 & & & & & & & 16.74 & 16.40 & 0.34 \\
\hline S1(INOV,STOV) & $26.04 .2006,29.04 .2006$ & $12 \quad 12 \quad 28.97$ & $+5343 \quad 36.4$ & 19.49 & 18.48 & 1.01 & 19.49 & 18.48 & 1.01 & & & \\
\hline S2(INOV,STOV) & $26.04 .2006,29.04 .2006$ & 121146.56 & +534026.9 & 18.92 & 17.94 & 0.98 & 18.92 & 17.94 & 0.98 & & & \\
\hline $\mathrm{J} 123743.1+630144.9$ & & 123743.09 & +630144.9 & 20.14 & 19.11 & 1.03 & & & & & & \\
\hline S1(INOV) & 27.04 .2006 & 123745.36 & +630059.3 & 20.49 & 18.92 & 1.57 & & & & & & \\
\hline S2(INOV) & 27.04 .2006 & $1237 \quad 55.04$ & +630648.7 & 19.44 & 18.94 & 0.50 & & & & & & \\
\hline S3(INOV) & 30.04 .2006 & $12 \quad 38 \quad 02.89$ & +630318.3 & 19.34 & 18.72 & 0.62 & & & & & & \\
\hline S4(INOV) & 30.04 .2006 & $1238 \quad 36.86$ & +630631.2 & 18.87 & 18.27 & 0.60 & & & & & & \\
\hline S5(STOV,LTOV) & $27.04 .2006^{\dagger}, 30.04 .2006^{\dagger}, 11.05 .2016^{*}$ & $1238 \quad 00.29$ & +630226.4 & & & & 17.51 & 16.87 & 0.64 & 17.51 & 16.87 & 0.64 \\
\hline S6(STOV,LTOV) & $27.04 .2006^{\dagger}, 30.04 .2006^{\dagger}, 11.05 .2016^{*}$ & 123730.63 & +630251.5 & & & & 18.72 & 17.98 & 0.74 & 18.72 & 17.98 & 0.74 \\
\hline J124225.3+642919.0 & & $1242 \quad 25.39$ & +642919.0 & 16.28 & 15.69 & 0.59 & & & & & & \\
\hline S2(INOV,STOV) & $25.04 .2006,28.04 .2006$ & $1242 \quad 17.56$ & +642532.7 & 17.93 & 16.92 & 1.01 & 17.93 & 16.92 & 1.01 & & & \\
\hline J133219.6+622715.9 & & 133219.65 & $+62 \quad 27 \quad 15.9$ & 19.50 & 19.18 & 0.32 & & & & & & \\
\hline S1(INOV) & 15.03 .2007 & $13 \quad 32 \quad 17.85$ & $+62 \quad 27 \quad 10.3$ & 19.98 & 18.51 & 1.47 & & & & & & \\
\hline S2(INOV) & 15.03 .2007 & 133159.40 & +623028.7 & 18.12 & 17.88 & 0.24 & & & & & & \\
\hline S3(INOV,STOV) & $15.03 .2007^{\dagger}, 16.03 .2007$ & 133154.55 & $+62 \quad 2239.9$ & 20.57 & 19.28 & 1.29 & 20.57 & 19.28 & 1.29 & & & \\
\hline S4(INOV,STOV) & $15.03 .2007^{\dagger}, 16.03 .2007$ & $13 \quad 32 \quad 30.17$ & +622838.0 & 19.31 & 18.90 & 0.41 & 19.31 & 18.90 & 0.41 & & & \\
\hline S1(LTOV) & $16.03 .2007^{\dagger}, 05.01 .2016^{*}$ & $\begin{array}{lll}13 & 32 & 17.85\end{array}$ & $+6227 \quad 10.3$ & & & & & & & 19.98 & 18.51 & 1.47 \\
\hline S5(LTOV) & $16.03 .2007^{\dagger}, 05.01 .2016^{*}$ & $1332 \quad 21.56$ & +622903.3 & & & & & & & 18.23 & 17.70 & 0.53 \\
\hline $\mathrm{J} 140923.9+261821.1$ & & 140923.90 & $+2618 \quad 21.1$ & 15.81 & 15.74 & 0.07 & & & & & & \\
\hline S1(INOV,LTOV) & $27.05 .2016,02.05 .2016^{*}$ & 140931.31 & +261749.6 & 16.27 & 15.72 & 0.55 & & & & 16.27 & 15.72 & 0.55 \\
\hline S2(INOV,LTOV) & $27.05 .2016,02.05 .2016^{*}$ & 140959.12 & +261426.4 & 15.73 & 15.22 & 0.51 & & & & 15.73 & 15.22 & 0.51 \\
\hline J153259.9-003944.1 & & 153259.96 & $\begin{array}{lll}-00 & 39 & 44.1\end{array}$ & 23.78 & 21.16 & 2.62 & & & & & & \\
\hline S1(INOV,STOV) & $\begin{array}{l}11.04 .2005,12.04 .2005^{*} \\
27.03 .2017,29.03 .2017^{*}\end{array}$ & 153243.66 & -004342.4 & 19.43 & 19.12 & 0.31 & 19.43 & 19.12 & 0.31 & & & \\
\hline S2(INOV,STOV) & $\begin{array}{l}\text { 11.04.2005,12.04.2005* } \\
27.03 .2017,29.03 .2017^{*}\end{array}$ & 153244.45 & $-0043 \quad 37.2$ & 18.89 & 18.79 & 0.10 & 18.89 & 18.79 & 0.10 & & & \\
\hline $\mathrm{J} 232428.4+144324.4$ & & $2324 \quad 28.43$ & +144324.4 & 19.04 & 18.77 & 0.27 & & & & & & \\
\hline S1(INOV) & 01.12 .2005 & 232438.42 & +144635.6 & 20.11 & 18.84 & 1.27 & & & & & & \\
\hline S2(INOV) & 01.12 .2005 & 232431.08 & +144725.4 & 20.07 & 18.64 & 1.43 & & & & & & \\
\hline S3(LTOV) & $01.12 .2005^{\dagger}, 10.10 .2016^{*}$ & $2324 \quad 28.79$ & +144229.7 & & & & & & & 17.77 & 16.93 & 0.84 \\
\hline
\end{tabular}

$a$ The DLCs obtained on dates marked by ' $*$ ' have been used only to look for STOV/LTOV, since they are either too noisy or insufficiently long $(\mathrm{T}<3 \mathrm{hr}$ ) for the purpose of INOV search. The ' $\dagger$ ' marks the dates whose WLQ DLCs using the comparison stars adopted for STOV/LTOV search are not shown in Fig. 1, as the DLCs used for INOV search on the date are already in Fig. 1, with the difference that the comparison stars used are not the same (more optimally selected in the case of INOV search, as described in Sect. 3)

software are systematically too low by a factor $\eta$ ranging between 1.3 and 1.75, as estimated in independent studies (e.g., Gopal-Krishna et al. 1995; Garcia et al. 1999; Sagar et al. 2004; Stalin et al. 2004b; Bachev et al. 2005). Recently, using a large sample, Goyal et al. (2013) estimated the best-fit value of $\eta$ to be 1.5 , which is adopted here. Thus, the $F^{\eta}-$ statistics can be expressed as:

$F_{1}^{\eta}=\frac{\sigma_{(q-s 1)}^{2}}{\eta^{2}\left\langle\sigma_{q-s 1}^{2}\right\rangle}, \quad F_{2}^{\eta}=\frac{\sigma_{(q-s 2)}^{2}}{\eta^{2}\left\langle\sigma_{q-s 2}^{2}\right\rangle}$,

where $\sigma_{(q-s 1)}^{2}$ and $\sigma_{(q-s 2)}^{2}$ are the variances of the 'quasar-star1' and 'quasar-star2' DLCs, with $\left\langle\sigma_{q-s 1}^{2}\right\rangle=$ $\sum_{\mathbf{i}=\mathbf{0}}^{N} \sigma_{i, e r r}^{2}(q-s 1) / N$ and $\left\langle\sigma_{q-s 2}^{2}\right\rangle=\sum_{\mathbf{i}=\mathbf{0}}^{N} \sigma_{i, e r r}^{2}(q-s 2) / N$ being the mean square (formal) rms errors of the individual data points in the 'quasar-star1' and 'quasar-star2' DLCs, respectively. Note that the number of points $(\mathrm{N})$ in all the DLCs for a given session is practically the same and the scaling factor $\eta$ is taken to be $=1.5$, as mentioned above.

The $F^{\eta}$-test is applied to a given 'quasar-star' DLC by computing its $F$ value using Eq. 1, and then comparing it with the critical value, $F_{\nu_{q s}}^{(\alpha)}$, where $\alpha$ is the significance level set for the test, and $\nu_{q s}$ is the degree of freedom $(\mathrm{N}-1)$ of the DLC. The two values we have set for the significance level are $\alpha=0.01$ and 0.05 , which correspond to confidence levels of greater than 99 and 95 per cent, respectively. If the computed $F$ value exceeds the corresponding critical value $F_{c}$, the null hypothesis (i.e., no variability) is discarded to the respective level of confidence. Thus, we denote a science target as variable (' $\mathrm{V}$ ') in a given session, if the computed $F$ values for both its DLCs are $\geqslant F_{c}(0.99)$, which corresponds to a confidence level $\geqslant 99$ per cent, and term it as nonvariable ('NV') if either of the two DLCs is found to have an $F$-value $\leqslant F_{c}(0.95)$. The remaining cases are classified as probably variable ('PV').

The inferred INOV status of the DLCs of each WLQ, relative to its two chosen comparison stars, are presented in Table 3 for each monitoring session. In the first 4 columns, we list the name of the WLQ, the date and duration of its monitoring and the number of data points $(\mathrm{N})$ (which is the same for both the DLCs of the WLQ). The next two columns list the computed $F$-values for the two 'quasar-star' DLCs and their INOV status, based on the $F^{\eta}$-test. Column 7 gives our averaged photometric error $\sigma_{i, e r r}(q-s)$ of the 
data points in the two 'quasar-star' DLCs. Typically, it is 0.03-mag for these relatively faint objects.

\section{RESULTS}

As seen from Table 2, we have monitored the 8 WLQs in the present sample on 20 nights. Of these, 7 nights' data could only be used to look for day-like, or long-term variability, since either the individual data points are too noisy (4 nights), or the DLCs are of insufficient duration $(\mathrm{T}<3$ hr) for the purpose of INOV search.

\subsection{Remarks on possible INOV events}

DLCs derived for the 19 intra-night monitoring sessions covering the 8 WLQs are displayed in Fig. 1, except the brief session of J153259.9-003944.1 on 29-03-2017 with DFOT which gave just two points and which is hence used only for STOV search. The top of each panel provides information on the telescope used, duration of the DLCs, and the type of variability searched using those DLCs. Among the 13 sessions used for INOV search, the telescope HCT was used for 5 of the sessions, OHP for 6 sessions and DFOT for 2 session. In each panel, the upper plot shows the star-star DLC for that session, while the lower two plots are DLCs of the WLQ relative to the two (comparison) stars. Note that, except for J153259.9-003944.1, the faintest object in the present sample (Table 1), at least one of the two comparison stars is within a magnitude of the WLQ monitored. The rms noise on individual data points in the DLCs varies from session to session and even within a session, depending on the changing observing conditions, such as the varying sky brightness due to the Moon, etc. Nonetheless, barring the case of WLQ J153259.9-003944.1, the typical $\mathrm{rms}$ noise of individual data points is $0.03 \mathrm{mag}$ (Table 3). Although, based on the statistical test mentioned in Sect. 4, there is no formal detection of INOV during any of the 13 sessions (Table 3 ), a visual inspection of the DLCs in Fig. 1 provides a reasonable hint of INOV in the following two sessions.

$\mathbf{J 1 0 4 8 3 3 . 5 6 + 6 2 0 3 0 5 . 0 ( 0 2 - 1 2 - 2 0 0 5 ) : ~ F o r ~ t h e ~ f i r s t ~ h a l f ~}$ an hour, the dip seen in the upper two panels (q-s2 and s1-s2 DLCs) is clearly due to variation of the star S2. The most prominent feature of the DLCs is an $\approx 0.1$-mag spike at $\sim 21.6 \mathrm{UT}$, in both q-s1 and q-s2 DLCs, while the s1-s2 DLC is steady over that time span. Here it is pertinent to note that although a photometric redshift $(z \sim 1.54)$ has been reported for this source (Richards et al. 2009) its extragalactic nature remains uncertain. This is, firstly, in view of a hint of proper motion (Table 1), but more so because of the lack of any features in its optical spectrum (e.g., see Plotkin et al. 2010a; Kügler et al. 2014a). For optical polarization of this source, Smith et al. (2007) have given an upper limit of $2.33 \%$. Clearly, a proper interpretation of the INOV results for this source remains contingent upon the availability of a secure measurements of its redshift and/or proper motion. Furthermore, the radio-optical spectral index $\alpha_{r-o} \quad(<0.27$, Collinge et al. 2005) for this source falls a bit short of the usually accepted upper limit of 0.21 for radio-quiet quasars (e.g., Luo et al. 2015). Therefore, in order to be certain of its radio-quiet classification, radio flux measurement is needed with a detection threshold a few times lower than attained in the FIRST survey (Becker et al. 1995), which is currently the deepest radio measurement available for this source.

J133219.65+622715.9 (15-03-2007): For this highredshift WLQ ( $z=3.1514$, Table 1) Collinge et al. (2005) give $\alpha_{r-o}=0.19$, which places it near the borderline between radio-quiet and radio-loud quasars. Indeed, with a radio-louness parameter $\mathrm{R}=33$ (Lane et al. 2011), this distant WLQ qualifies to be a "Radio Intermediate Quasar (RIQ)" (e.g., Miller et al. 1993; Falcke et al. 1995, 1996; Barvainis et al. 2005). A well known local prototype of this class is III Zw 2 which is known to exhibit a blazar-like behaviour, i.e., a large radio variability (Teraesranta et al. 1998), a superluminal motion (Brunthaler et al. 2000, 2005 ), as well as $\gamma$-ray flaring in the radio core (Liao et al. 2016). In fact, based on rapid radio flux variability, relativistically beamed jets are inferred to be common among RIQs (Wang et al. 2006). However, a study of the optical variability of RIQs on hour to day-like time scales, based on intranight monitoring of 10 RIQs on 42 nights, has shown that while intra-night and inter-night optical variability is exhibited by RIQs, the INOV amplitude hardly ever exceeds $3-4 \%$, in clear contrast to blazars (Goyal et al. 2010). This is consistent with the inference that a relativistically beamed radio core of a quasar is not a sufficient condition for observing a pronounced INOV (Goyal et al. 2012).

Broadly, the trend visually inferred from each DLC of this WLQ is a $\sim 0.1-\mathrm{mag}$ brightening over the 5 hour session on 15.03.2007, relative to both comparison stars which themselves remained steady (Fig. 1; Table 3). Additional information on rapid optical variability of this source is provided in Sect. 6.1. It should be mentioned that the source was found to be unpolarised $(\mathrm{p}<1.7 \%)$ in the optical, which corresponds to UV in the rest-frame (Heidt \& Nilsson 2011).

\subsection{Notes on inter-day variability events}

For 5 WLQs in the present sample, the observations reported here also enable us to investigate STOV on day-like time scale (Table 2). Each of these sources was monitored on two epochs separated by 1 to 3 days. We emphasize that for computing the STOV (or LTOV) status of a WLQ, as reported in Table 4, we have used the same pair of comparison stars for all the monitoring sessions. In each case, the stars were found to remain steady, but the session-averaged brightness showed a small, yet significant change which is $0.035 \pm 0.008 \mathrm{mag}$ for $\mathrm{J} 121221.5+534128.0$; $0.043 \pm 0.009$ for $\mathrm{J} 133219.6+622715.9$ and $0.039 \pm 0.013 \& 0.223 \pm 0.083$ for J153259.9-003944.1 in the two STOV sessions (Table 4). Applying the F-test on the combined DLCs, we found that only J133219+622715 shows variability at more than $99 \%$ confidence level. This is further discussed in Sect. 6 .

\subsection{Variability on month/year-like time scales}

For 5 of our WLQs, the observations reported here permit investigation of LTOV (see Table 2). As seen from Table 4, LTOV is clearly witnessed for J104833.5+620305.0, 

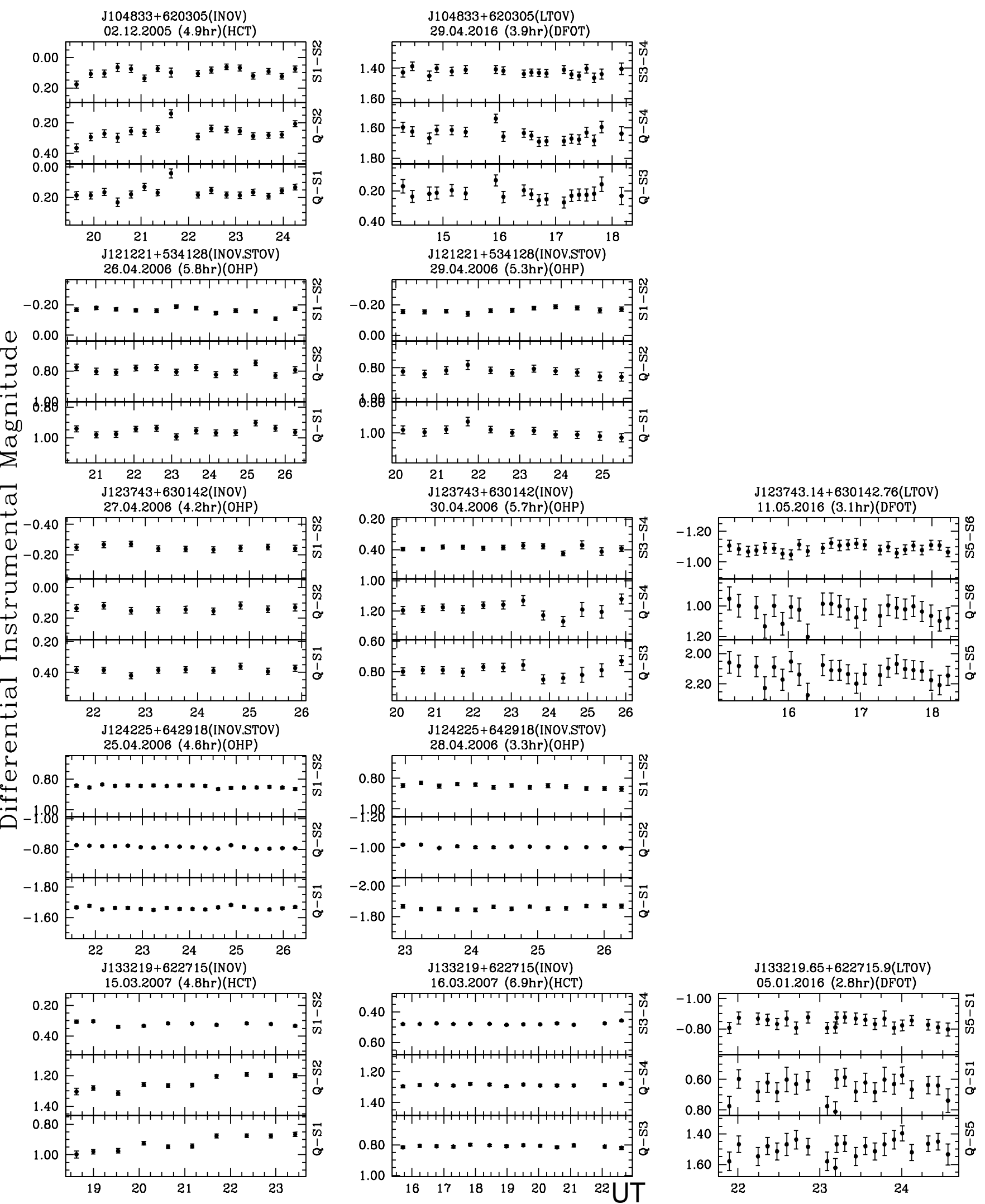

Figure 1. Differential light curves (DLCs) in R-band, for the 8 WLQs in our sample. Name of the WLQ along with the date and duration of its monitoring session, are given at the top of each panel together with the telescope's name and the type of variability for which the DLCs were used. Within each panel the uppermost DLC is derived using the light curves of the two comparison stars, while the lower two DLCs are the light curves of the WLQ relative to the two comparison stars, as defined in the labels just outside the panel on the right side. (cont.) 
Table 3. Observational details and the inferred INOV status for the present sample of 8 WLQs monitored in 13 sessions.

\begin{tabular}{|c|c|c|c|c|c|c|}
\hline RQWLQ & $\begin{array}{c}\text { Date } \\
\text { dd.mm.yyyy } \\
(2)\end{array}$ & $\begin{array}{l}\mathrm{T} \\
\mathrm{hr} \\
(3)\end{array}$ & $\mathrm{N}$ & $\begin{array}{r}\text { F-test values } \\
F_{1}^{\eta}, F_{2}^{\eta} \\
(5)\end{array}$ & $\begin{array}{r}\text { INOV status } \\
\mathrm{F}_{\eta} \text {-test } \\
(6)\end{array}$ & $\begin{array}{r}\sqrt{\left\langle\sigma_{i, e r r}^{2}\right\rangle} \\
(\mathrm{q}-\mathrm{s}) \\
(7)\end{array}$ \\
\hline $\mathrm{J} 104833.5+620305.0$ & 02.12 .2005 & 4.95 & 17 & $1.12,1.42$ & NV,NV & 0.04 \\
\hline J121221.5+534128.0 & 26.04 .2006 & 5.78 & 12 & $0.71,0.64$ & $\mathrm{NV}, \mathrm{NV}$ & 0.03 \\
\hline J121221.5+534128.0 & 29.04 .2006 & 5.28 & 11 & $0.24,0.34$ & $\mathrm{NV}, \mathrm{NV}$ & 0.04 \\
\hline $\mathrm{J} 123743.1+630144.9$ & 27.04 .2006 & 4.20 & 9 & $0.30,0.16$ & NV,NV & 0.03 \\
\hline $\mathrm{J} 123743.1+630144.9$ & 30.04 .2006 & 5.73 & 12 & $0.76,0.63$ & $\mathrm{NV}, \mathrm{NV}$ & 0.05 \\
\hline $\mathrm{J} 124225.3+642919.0$ & 25.04 .2006 & 4.65 & 18 & $0.64,1.56$ & $\mathrm{NV}, \mathrm{NV}$ & 0.01 \\
\hline $\mathrm{J} 124225.3+642919.0$ & 28.04 .2006 & 3.29 & 13 & $0.88,1.25$ & $\mathrm{NV}, \mathrm{NV}$ & 0.01 \\
\hline J133219.6+622715.9 & 15.03 .2007 & 4.79 & 10 & $5.24,4.84$ & PV,PV & 0.03 \\
\hline J133219.6+622715.9 & 16.03 .2007 & 6.90 & 13 & $0.59,0.49$ & $\mathrm{NV}, \mathrm{NV}$ & 0.02 \\
\hline $\mathrm{J} 140923.9+261821.1$ & 27.05 .2016 & 3.66 & 40 & $0.37,0.54$ & $\mathrm{NV}, \mathrm{NV}$ & 0.01 \\
\hline J153259.9-003944.1 & 11.04 .2005 & 4.50 & 15 & $0.44,0.48$ & $\mathrm{NV}, \mathrm{NV}$ & 0.08 \\
\hline J153259.9-003944.1 & 27.03 .2017 & 4.65 & 10 & $1.49,1.59$ & $\mathrm{NV}, \mathrm{NV}$ & 0.08 \\
\hline $\mathrm{J} 232428.4+144324.4$ & 01.12 .2005 & 2.92 & 10 & $0.81,1.04$ & $\mathrm{NV}, \mathrm{NV}$ & 0.02 \\
\hline
\end{tabular}

$a \mathrm{~V}=$ variable, i.e., confidence level $\geqslant 0.99$ for both DLCs; PV=probable variable, i.e., $0.95-0.99$ confidence level for both DLCs; $\mathrm{NV}=$ non-variable, i.e., confidence level $<0.95$ for one or both DLCs. Variability status inferred using the quasar-star1 and quasar-star2 pairs are separated by a comma.

$\mathrm{J} 123743.1+630144.9$ and $\mathrm{J} 232428.4+144324.4$, relative to both their comparison stars. Unfortunately, the large variability of $1.655 \pm 0.009$ mag observed for J104833.5+620305.0 is difficult to interpret at present, since extragalactic nature of this source remains to be established (Sect. 5.1). Thus, in the present sample only two sources viz, $\mathrm{J} 123743.1+630144.9(z=3.4278)$ and $\mathrm{J} 232428.4+144324.4$ $(z=1.4172)$ showing level changes over a decade of $0.163 \pm 0.010 \mathrm{mag}$ and $0.144 \pm 0.018 \mathrm{mag}$, respectively, are at present clear examples of a radio-quiet WLQ displaying a substantial long-term variability. A previously known such example is J153259.9-003944.1 $(z=4.62)$ for which $i$ - band photometry spanning 7-years has revealed a variability of amplitude $\sim 0.1-\mathrm{mag}$ (Diamond-Stanic et al. 2009). Note that for the RQWLQs J153259.9-003944.1 and J123743.1+630144.9, Lane et al. (2011) have presented SEDs covering the rest-frame mid-IR to UV, and have inferred them to be fully consistent with radio-quiet quasars and displaying no features characteristic of BL Lacs.

\section{DISCUSSION AND CONCLUSIONS}

From early on it has been recognized that a multi-pronged probe is the key to unraveling the nature and composition of the WLQ population which is small but continues to be enigmatic (e.g., Wu et al. 2011). The strategies employed include measurement of multi-wavelength spectral, as well as polarimetric and temporal properties. Our investigations are focused on the last tool, namely the temporal properties of the broad-band optical continuum, going down to hour-like time scale. The present study reports intranight differential light curves (DLCs) for 8 RQWLQs monitored in 20 sessions, longer than 3 hours (barring the three sessions on 02-05-2016, 10-10-2016 and 29-03-2017 devoted to the RQWLQs J140923.9+261821.1, J232428.4+144324.4 and J153259.9-003944.1, respectively, which lasted for just $\sim 1.5$ hour (or even less) and hence used for STOV/LTOV search only, (see Fig. 1 and Table 2). Out of the 8 radioquiet/weak WLQs, six have been classified as 'radio-quiet probable BL Lacs' by Collinge et al. (2005), while the remaining two are reported to have exhibited an episode of large, blazar-like optical variability (Sect. 2). These two RQWLQs are: J140923.9+261821.1 (Blundell et al. 2003) and J153259.9-003944.1 (Stalin \& Srianand 2005). As in Papers I - IV, an INOV detection threshold of $\sim 0.1-$ mag was typically achieved for 13 of our intra-night sessions of $>3$ hours, covering these 8 sources (Table 3). As mentioned in Sect. 5, even though, based on formal statistical test, none of the 13 sessions exhibited INOV, a visual inspection does provide a hint of INOV in two of the sessions, namely on 02-12-2005 (J104833.5+620305.0) and on 15-03-2007 (J133219.6+622715.9). Interestingly, the J133219.6+622715.9 is a high redshift 'radio-intermediate' WLQ and the same classification cannot at present be excluded even for the J104833.5+620305.0, given that the existing upper limit to its radio flux falls a few times short of that needed to confirm radio quietness (Sect. 5.1). Furthermore, the extragalactic classification of J104833.5+620305.0 remains unestablished, since no feature has been detected in its optical spectra, thus underscoring the need for a deeper spectroscopy (Sect. 5.1). The low rate of INOV detection for the present sample of WLQs is in conformity with our previously published INOV results for an independent, larger sample of RQWLQs for which just two incidences of INOV were found (Papers I to IV; Sect. 1).

For five members of our sample of radio-quiet/weak WLQs, the present multi-epoch intra-night monitoring has also enabled a sensitive search for STOV on day-like time scale, and LTOV on month/year-like time scale. The present study of STOV/LTOV has the merit that for a given WLQ, the DLCs of the intranight sessions being compared were derived using the same comparison stars. Also, for each WLQ, either both comparison stars were found to have the same brightness in the two sessions, or at least one of the stars 

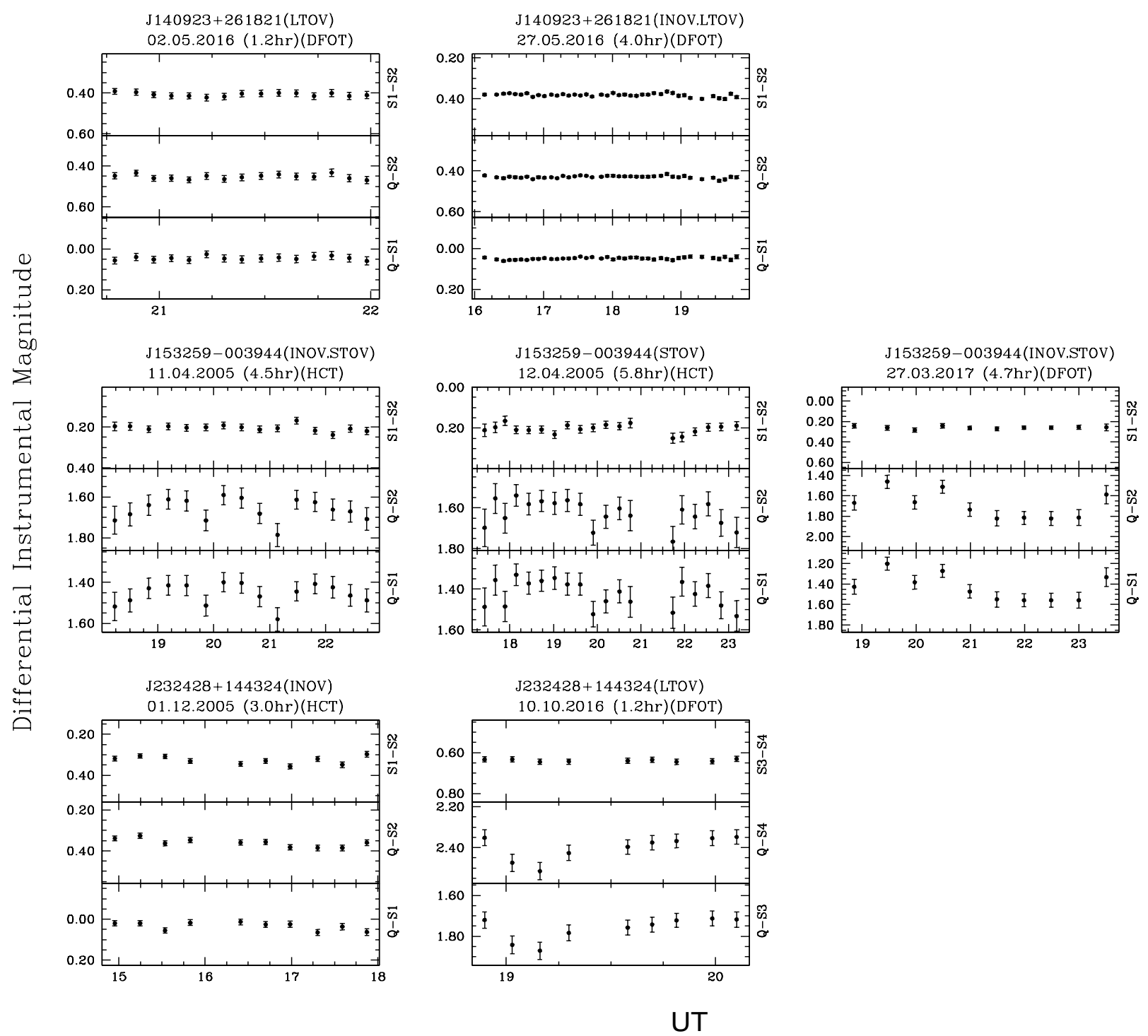

Figure 1 - continued

could be clearly identified as the steady one. The results are summarized below.

\subsection{Short-term Optical Variability on day-like time scale}

For the 3 of the 5 WLQs, the observed difference between the session-averaged brightness levels show a statistically significant STOV, the variation on day-like time scale being $0.035 \pm 0.008 \mathrm{mag}$ for $\mathrm{J} 121221.5+534128.0 ; 0.043 \pm 0.009$ mag for J133219.6+622715.9; and $0.039 \pm 0.013 \mathrm{mag} \&$ $0.223 \pm 0.083 \mathrm{mag}$ for J153259.9-003944.1 ( Table 4). In the rest-frame, the time scale for these 4 STOV events lie between 5 and 18 hours. While, as mentioned above, $\mathrm{J} 133219.6+622715.9$ is a radio-intermediate type, the other two WLQs are bona-fide RQWLQs, (Table 1). It is instructive to compare the inter-day variability rate of these 3
WLQs with the ensemble variability of optically selected quasars (i.e., overwhlemingly RQQs) on day-like time scale. Such a 'control sample' of typical quasars can, for instance, be built using the analysis of the $R$-band monitoring of 28,000 luminous, broad-line AGNs from the SDSS, under the 'intermediate Palomar Transient Factory' (iPTF) and the 'Palomar Transient Factory' (PTF) surveys (Caplar et al. 2017). Using this huge dataset, these authors have shown that the amplitude of variability exhibits a clear anti-correlation with luminosity and little or no variation with black-hole mass or redshift. Further, for the (radio-quiet) QSOs in the highest redshift bin $(<\mathrm{z}\rangle$ $=2.17$ ) of their sample (which approach fairly closely our 3 WLQs in luminosity), they find a variability amplitude of at most $1-2 \%$ on 1 -day time scale in the rest-frame (section 4.1 of their paper). This is consistent with the restframe value of $\sim 1 \%$ estimated for intra-day optical variabil- 
Table 4. Session-averaged brightness (in unit of instrumental magnitude) of the DLCs of the 8 WLQs and their comparison stars on two epochs.

\begin{tabular}{|c|c|c|c|}
\hline $\begin{array}{c}\text { Source Name } \\
\text { (variability type) }\end{array}$ & Averaged brightness(epoch 1,Telescope) & Averaged brightness(epoch 2, Telescope) & Brightness change (mag) \\
\hline \multicolumn{4}{|l|}{ J104833.5+620305.0 } \\
\hline Q-S4(LTOV) & $3.275 \pm 0.008(02.12 .2005, \mathrm{HCT})$ & $1.641 \pm 0.009(29.04 .2016, \mathrm{DFOT})$ & $-1.634 \pm 0.012$ \\
\hline S3-S4(LTOV) & $1.382 \pm 0.001(02.12 .2005, \mathrm{HCT})$ & $1.424 \pm 0.004(29.04 .2016, \mathrm{DFOT})$ & $0.042 \pm 0.004$ \\
\hline \multicolumn{4}{|l|}{$\mathrm{J} 121221.5+534128.0$} \\
\hline Q-S1(STOV) & $0.955 \pm 0.007(26.04 .2006, \mathrm{OHP})$ & $0.992 \pm 0.007(29.04 .2006, \mathrm{OHP})$ & $0.037 \pm 0.009$ \\
\hline \multicolumn{4}{|l|}{$\mathrm{J} 123743.1+630144.9$} \\
\hline Q-S5(STOV) & $2.305 \pm 0.004(27.04 .2006, \mathrm{OHP})$ & $2.300 \pm 0.008(30.04 .2006, \mathrm{OHP})$ & $-0.005 \pm 0.008$ \\
\hline Q-S6(STOV) & $1.186 \pm 0.004(27.04 .2006, \mathrm{OHP})$ & $1.186 \pm 0.002(30.04 .2006, \mathrm{OHP})$ & $0.000 \pm 0.012$ \\
\hline S5-S6(STOV) & $-1.118 \pm 0.003(27.04 .2006, \mathrm{OHP})$ & $-1.110 \pm 0.006(30.04 .2006, \mathrm{OHP})$ & $0.008 \pm 0.006$ \\
\hline Q-S5(LTOV) & $2.300 \pm 0.008(27.04 .2006, \mathrm{OHP})$ & $2.126 \pm 0.010(11.05 .2016, \mathrm{DFOT})$ & $-0.174 \pm 0.012$ \\
\hline Q-S2(STOV) & $-1.035 \pm 0.002(25.04 .2006, \mathrm{OHP})$ & $-1.034 \pm 0.001(28.04 .2006, \mathrm{OHP})$ & $0.001 \pm 0.002$ \\
\hline S1-S2(STOV) & $0.851 \pm 0.003(25.04 .2006, \mathrm{OHP})$ & $0.851 \pm 0.003(28.04 .2006, \mathrm{OHP})$ & $0.000 \pm 0.004$ \\
\hline \multicolumn{4}{|l|}{ J133219.6+622715.9 } \\
\hline $\mathrm{Q}-\mathrm{S} 3(\mathrm{STOV})$ & $0.767 \pm 0.012(15.03 .2007, \mathrm{HCT})$ & $0.808 \pm 0.001(16.03 .2007, \mathrm{HCT})$ & $0.041 \pm 0.012$ \\
\hline Q-S4(STOV) & $1.241 \pm 0.013(15.03 .2007, \mathrm{HCT})$ & $1.286 \pm 0.001(16.03 .2007, \mathrm{HCT})$ & $0.045 \pm 0.013$ \\
\hline S3-S4(STOV) & $0.473 \pm 0.003(15.03 .2007, \mathrm{HCT})$ & $0.478 \pm 0.001(16.03 .2007, \mathrm{HCT})$ & $0.005 \pm 0.003$ \\
\hline Q-S5(LTOV) & $1.466 \pm 0.001(16.03 .2007, \mathrm{HCT})$ & $1.492 \pm 0.009(05.01 .2016, \mathrm{DFOT})$ & $0.026 \pm 0.009$ \\
\hline Q-S1(LTOV) & $0.808 \pm 0.001(16.03 .2007, \mathrm{HCT})$ & $0.655 \pm 0.011(05.01 .2016, \mathrm{DFOT})$ & $-0.153 \pm 0.011$ \\
\hline S5-S1(LTOV) & $-0.658 \pm 0.001(16.03 .2007, \mathrm{HCT})$ & $-0.836 \pm 0.005(05.01 .2016, \mathrm{DFOT})$ & $-0.178 \pm 0.005$ \\
\hline \multicolumn{4}{|l|}{$\mathrm{J} 140923.9+261821.1$} \\
\hline $\mathrm{Q}-\mathrm{S} 1(\mathrm{LTOV})$ & $0.045 \pm 0.002(02.05 .2016, \mathrm{DFOT})$ & $0.048 \pm 0.001(27.05 .2016, \mathrm{DFOT})$ & $0.003 \pm 0.002$ \\
\hline $\mathrm{Q}-\mathrm{S} 2(\mathrm{LTOV})$ & $0.453 \pm 0.002(02.05 .2016, \mathrm{DFOT})$ & $0.429 \pm 0.001(27.05 .2016, \mathrm{DFOT})$ & $-0.024 \pm 0.002$ \\
\hline S1-S2(STOV) & $0.260 \pm 0.003(27.03 .2017, \mathrm{DFOT})$ & $0.244 \pm 0.028(29.03 .2017, \mathrm{DFOT})$ & $-0.016 \pm 0.028$ \\
\hline \multicolumn{4}{|l|}{$\mathrm{J} 232428.4+144324.4$} \\
\hline Q-S3(LTOV) & $1.624 \pm 0.003(01.12 .2005, \mathrm{HCT})$ & $1.757 \pm 0.017(10.10 .2016, \mathrm{DFOT})$ & $0.133 \pm 0.017$ \\
\hline Q-S4(LTOV) & $2.240 \pm 0.002(01.12 .2005, \mathrm{HCT})$ & $2.394 \pm 0.018(10.10 .2016$, DFOT $)$ & $0.154 \pm 0.018$ \\
\hline S3-S4(LTOV) & $0.616 \pm 0.001(01.12 .2005, \mathrm{HCT})$ & $0.637 \pm 0.001(10.10 .2016$, DFOT $)$ & $0.021 \pm 0.001$ \\
\hline
\end{tabular}

ity of radio-quiet QSOs in the LINEAR survey (Ruan et al. 2012). These variability amplitudes for typical radio-quiet QSOs are somewhat smaller, although probably not inconsistent with the values given above for the 3 WLQs, namely $\mathrm{J} 121221.5+534128.0(z=3.0976), \mathrm{J} 133219.6+622715.9(z=$ $3.1514)$ and J153259.9-003944.1 $(z=4.62)$. Also, note that for blazars, Ruan et al. (2012) give a typical intra-night amplitude of $\sim 5 \%$ (see above), very similar to the typical value evident from the INOV amplitudes reported by Goyal et al. (2013) for a large sample of 24 blazars monitored in 85 sessions of around 6 hour duration each. Still, in our view, it would be pre-mature to associated the observed (rest-frame) intra-day variability of these 3 high- $z$ WLQs with the blazar phenomenon particularly because the variability is intrinsically in the far-UV band. This is relevant since normal
QSOs are found to be distinctly more variable in the restframe UV, and even more in the far-UV (Welsh et al. 2011; Edelson et al. 1996; Paltani et al. 1998; Collier et al. 2001). Conceivably, the larger UV variations are driven by X-ray flares above the inner accretion disk, which are processed inside the disk (e.g., Rokaki et al. 1993; Wiita 1996; Liu et al. 2016, and references therein).

Finally, turning to longer time scales, the present observations have enabled a comparison of optical brightness at rest-frame time intervals between 2 to 5 years for four of our WLQs and of $\sim 2$ weeks for J140923.9+261821.1 (Table 4). Down to 2-3\% limit, no long-term variability was detected for the radio-intermediate WLQ J133219.6+622715.9, and the well known radio-quiet WLQ J140923.9+261821.1 (Sect. 2; Table 1). In contrast, the other 
3 WLQs, namely J104833.5+620305.0, J123743.1+630144.9 and J232428.4+144324.4 were found to vary prominently over a rest-frame time interval of $\sim 4,2$ and 4 yr respectively (Table 4). In particular, the variability of $1.655 \pm 0.009$ mag exhibited by J104833.5+620305.0 appears blazar-like, since on such time scales, optical variability is mostly limited to $~ 0.4-$ mag even for BL Lacs belonging to optically selected samples; variability amplitudes above 1.5-mag are extremely rare (e.g., Kügler et al. 2014b). This makes it all the more important to ascertain the extragalactic as well as radio-quiet classification for this object through more sensitive optical spectroscopy and deeper radio measurements reaching a detection threshold of $\sim 0.1 \mathrm{mJy}$ (Sect. 5). As of now, the only two confirmed radio-quiet WLQ in our sample, for which a large long-term variability $(0.163 \pm 0.010 \mathrm{mag}$ and $0.144 \pm 0.018 \mathrm{mag}$, over a decade) is claimed here are J123743.1+630144.9 ( $z=3.4278)$ and J232428.4+144324.4 ( $z=1.1472)$, respectively. It is also pertinent to note that optical polarimetry of all these three sources, viz J104833.5+620305.0, J123743.1+630144.9 and J232428.4+144324.4, has yielded a low degree $(\mathrm{p}<\sim 2 \%$ ) of polarization (Smith et al. 2007; Heidt \& Nilsson 2011), which appears too modest to justify a BL Lac classification. However, strong polarization variability being the hallmark of BL Lacs, even bona-fide BL Lac objects may sometimes appear weakly polarised ( $p<3 \%$ ) (e.g., Wills et al. 1992). According to Fugmann (1988), there is $~ 40 \%$ chance that a bona-fide blazar will exhibit $p<3 \%$ at a random epoch. Likewise, in another, independent study it was shown that the fraction of time an X-ray selected BL Lac exhibits $p$ $>4 \%$ is only $\sim 50 \%$ (Jannuzi et al. 1994). Therefore, the single-epoch measurements of $p<\sim 2 \%$ do not rule out a BL Lac classification, per se (note that even the very prominent BL Lac object OJ 287 has been found unpolarized sometimes, e.g., see Villforth et al. (2009)). A multi-epoch optical polarimetry will clearly be needed to ascertain a BL Lac classification for these interesting objects.

\section{ACKNOWLEDGEMENTS}

We are very thankful to an anonymous referee for insightful comments on the manuscript. G-K is partly supported by a Platinum Jubilee Senior Scientist Fellowship of the National Academy of Sciences, India.

\section{REFERENCES}

Abazajian K. et al., 2004, AJ, 128, 502

Anderson S. F. et al., 2001, AJ, 122, 503

Anderson S. F. et al., 2007, AJ, 133, 313

Angel J. R. P., Stockman H. S., 1980, ARA\&A, 18, 321

Antonucci R., 2012, Astronomical and Astrophysical Transactions, 27, 557

Bachev R., Strigachev A., Semkov E., 2005, MNRAS, 358, 774

Barvainis R., Lehár J., Birkinshaw M., Falcke H., Blundell K. M., 2005, ApJ, 618, 108

Bauer A. et al., 2009, ApJ, 705, 46

Becker R. H., White R. L., Helfand D. J., 1995, ApJ, 450, 559
Begelman M. C., Blandford R. D., Rees M. J., 1984, Reviews of Modern Physics, 56, 255

Blandford R. D., Rees M. J., 1978, PhyS, 17, 265

Blundell K. M., Beasley A. J., 1998, MNRAS, 299, 165

Blundell K. M., Beasley A. J., Bicknell G. V., 2003, ApJ, 591, L103

Borra E. F., Corriveau G., 1984, ApJ, 276, 449

Boyle B. J., Shanks T., Croom S. M., Smith R. J., Miller L., Loaring N., Heymans C., 2000, MNRAS, 317, 1014

Brunthaler A., Falcke H., Bower G. C., Aller M. F., Aller

H. D., Teräsranta H., 2005, A\&A, 435, 497

Brunthaler A. et al., 2000, A\&A, 357, L45

Caccianiga A., Marchã M. J. M., Thean A., DennettThorpe J., 2001, MNRAS, 328, 867

Caplar N., Lilly S. J., Trakhtenbrot B., 2017, ApJ, 834, 111 Carini M. T., Miller H. R., Noble J. C., Goodrich B. D., 1992, AJ, 104, 15

Carini M. T., Miller H. R., Noble J. C., Sadun A. C., 1991, AJ, 101, 1196

Cellone S. A., Romero G. E., Combi J. A., 2000, AJ, 119, 1534

Chanan G. A., Downes R. A., Chance D., Margon B., Helfand D. J., 1982, ApJ, 261, L31

Chand H., Kumar P., Gopal-Krishna, 2014, MNRAS, 441, 726

Collier S. et al., 2001, ApJ, 561, 146

Collinge M. J. et al., 2005, AJ, 129, 2542

Croom S., Smith R., Boyle B., Shanks T., Loaring N., Miller L., 2001, Anglo-Australian Observatory Epping Newsletter, 97, 3

de Diego J. A., 2010, AJ, 139, 1269

Diamond-Stanic A. M. et al., 2009, ApJ, 699, 782

Edelson R. A. et al., 1996, ApJ, 470, 364

Falcke H., Malkan M. A., Biermann P. L., 1995, A\&A, 298, 375

Falcke H., Patnaik A. R., Sherwood W., 1996, ApJ, 473, L13

Fan X. et al., 1999, ApJ, 526, L57

Fan X. et al., 2006, AJ, 131, 1203

Fugmann W., 1988, A\&A, 205, 86

Gallo L. C., 2006, MNRAS, 365, 960

Garcia A., Sodré L., Jablonski F. J., Terlevich R. J., 1999, MNRAS, 309, 803

Gopal-Krishna, Joshi R., Chand H., 2013, MNRAS, 430, 1302

Gopal-Krishna, Sagar R., Wiita P. J., 1995, MNRAS, 274, 701

Goyal A., Gopal-Krishna, Joshi S., Sagar R., Wiita P. J., Anupama G. C., Sahu D. K., 2010, MNRAS, 401, 2622

Goyal A., Gopal-Krishna, Wiita P. J., Anupama G. C., Sahu D. K., Sagar R., Joshi S., 2012, A\&A, 544, A37

Goyal A., Gopal-Krishna, Wiita P. J., Stalin C. S., Sagar R., 2013, MNRAS, 435, 1300(GGWSS13)

Heidt J., Nilsson K., 2011, A\&A, 529, A162

Herrera Ruiz N., Middelberg E., Norris R. P., Maini A., 2016, A\&A, 589, L2

Hewett P. C., Wild V., 2010, MNRAS, 405, 2302

Hryniewicz K., Czerny B., Nikołajuk M., Kuraszkiewicz J., 2010, MNRAS, 404, 2028

Impey C. D., Brand P. W. J. L., 1982, MNRAS, 201, 849

Jang M., Miller H. R., 1997, AJ, 114, 565

Jannuzi B. T., Green R. F., French H., 1993, ApJ, 404, 100 
Jannuzi B. T., Smith P. S., Elston R., 1994, ApJ, 428, 130 Joshi R., Chand H., Gupta A. C., Wiita P. J., 2011, MNRAS, 412, 2717

Kellermann K. I., Sramek R., Schmidt M., Shaffer D. B., Green R., 1989, AJ, 98, 1195

Kügler S. D., Gianniotis N., Polsterer K. L., 2015, MNRAS, 451, 3385

Kügler S. D., Nilsson K., Heidt J., Esser J., Schultz T., 2014a, A\&A, 569, A95

Kügler S. D., Nilsson K., Heidt J., Esser J., Schultz T., 2014b, A\&A, 569, A95

Kumar P., Chand H., Gopal-Krishna, 2016, MNRAS, 461, 666

Kumar P., Gopal-Krishna, Chand H., 2015, MNRAS, 448, 1463

Lane R. A. et al., 2011, ApJ, 743, 163

Laor A., Davis S. W., 2011, MNRAS, 417, 681

Leighly K. M., Halpern J. P., Jenkins E. B., Grupe D., Choi J., Prescott K. B., 2007, ApJ, 663, 103

Leipski C., Falcke H., Bennert N., Hüttemeister S., 2006, A\&A, 455, 161

Liao N.-H., Xin Y.-L., Fan X.-L., Weng S.-S., Li S.-K., Chen L., Fan Y.-Z., 2016, ApJS, 226, 17

Liu H., Li S.-L., Gu M., Guo H., 2016, MNRAS, 462, L56

Londish D. et al., 2002, MNRAS, 334, 941

Londish D. et al., 2007, MNRAS, 374, 556

Luo B. et al., 2015, ApJ, 805, 122

McDowell J. C., Canizares C., Elvis M., Lawrence A., Markoff S., Mathur S., Wilkes B. J., 1995, ApJ, 450, 585 Meusinger H., Balafkan N., 2014, A\&A, 568, A114

Miller P., Rawlings S., Saunders R., 1993, MNRAS, 263, 425

Monet D. G. et al., 2003, AJ, 125, 984

Murray N., Chiang J., 1997, ApJ, 474, 91

Nicastro F., Martocchia A., Matt G., 2003, ApJ, 589, L13

Nikołajuk M., Walter R., 2012, MNRAS, 420, 2518

Padovani P., Giommi P., Landt H., Perlman E. S., 2007, ApJ, 662, 182

Paltani S., Courvoisier T. J.-L., Walter R., 1998, A\&A, 340, 47

Perlman E. S. et al., 1996, ApJS, 104, 251

Plotkin R. M. et al., 2010a, AJ, 139, 390

Plotkin R. M., Anderson S. F., Brandt W. N., DiamondStanic A. M., Fan X., MacLeod C. L., Schneider D. P., Shemmer O., 2010b, ApJ, 721, 562

Plotkin R. M., Anderson S. F., Hall P. B., Margon B., Voges W., Schneider D. P., Stinson G., York D. G., 2008, AJ, 135, 2453

Plotkin R. M. et al., 2015, ApJ, 805, 123

Prabhu T. P., Anupama G. C., 2010, in Astronomical Society of India Conference Series, Vol. 1, Astronomical Society of India Conference Series

Richards G. T. et al., 2009, ApJS, 180, 67

Rokaki E., Collin-Souffrin S., Magnan C., 1993, A\&A, 272, 8

Ruan J. J. et al., 2012, ApJ, 760, 51

Sagar R. et al., 2011, CURRENT-SCIENCE, 101, 8

Sagar R., Stalin C. S., Gopal-Krishna, Wiita P. J., 2004, MNRAS, 348, 176

Schneider D. P. et al., 2005, AJ, 130, 367

Shemmer O., Brandt W. N., Anderson S. F., Diamond-

Stanic A. M., Fan X., Richards G. T., Schneider D. P.,
Strauss M. A., 2009, ApJ, 696, 580

Shemmer O. et al., 2006, ApJ, 644, 86

Shemmer O. et al., 2010, ApJ, 722, L152

Smith P. S., Williams G. G., Schmidt G. D., DiamondStanic A. M., Means D. L., 2007, ApJ, 663, 118

Stalin C. S., Gopal-Krishna, Sagar R., Wiita P. J., 2004a, MNRAS, 350, 175

Stalin C. S., Gopal Krishna, Sagar R., Wiita P. J., 2004b, Journal of Astrophysics and Astronomy, 25, 1

Stalin C. S., Srianand R., 2005, MNRAS, 359, 1022

Stetson P. B., 1987, PASP, 99, 191

Stetson P. B., 1992, in Astronomical Society of the Pacific Conference Series, Vol. 25, Astronomical Data Analysis Software and Systems I, Worrall D. M., Biemesderfer C., Barnes J., eds., p. 297

Stickel M., Padovani P., Urry C. M., Fried J. W., Kuehr H., 1991, ApJ, 374, 431

Stocke J. T., 2001, in Astronomical Society of the Pacific

Conference Series, Vol. 227, Blazar Demographics and

Physics, Padovani P., Urry C. M., eds., p. 184

Stocke J. T., Morris S. L., Gioia I., Maccacaro T., Schild R. E., Wolter A., 1990, ApJ, 348, 141

Stocke J. T., Morris S. L., Gioia I. M., Maccacaro T., Schild R., Wolter A., Fleming T. A., Henry J. P., 1991, ApJS, 76,813

Teraesranta H. et al., 1998, A\&AS, 132, 305

Ulvestad J. S., Antonucci R. R. J., Barvainis R., 2005, ApJ, 621,123

Urry C. M., Padovani P., 1995, PASP, 107, 803

Villforth C., Koekemoer A. M., Grogin N. A., 2010, ApJ, 723,737

Villforth C., Nilsson K., Østensen R., Heidt J., Niemi S.M., Pforr J., 2009, MNRAS, 397, 1893

Wang T.-G., Zhou H.-Y., Wang J.-X., Lu Y.-J., Lu Y., 2006, ApJ, 645, 856

Welsh B. Y., Wheatley J. M., Neil J. D., 2011, A\&A, 527, A15

Wiita P. J., 1996, in Astronomical Society of the Pacific Conference Series, Vol. 110, Blazar Continuum Variability, Miller H. R., Webb J. R., Noble J. C., eds., p. 42

Wills B. J., Wills D., Breger M., Antonucci R. R. J., Barvainis R., 1992, ApJ, 398, 454

Wu J., Brandt W. N., Anderson S. F., Diamond-Stanic A. M., Hall P. B., Plotkin R. M., Schneider D. P., Shemmer O., 2012, ApJ, 747, 10

Wu J. et al., 2011, ApJ, 736, 28

York D. G. et al., 2000, AJ, 120, 1579 